\title{
Imaging and Spectroscopy with Focal Reducers
}

\author{
W. Seifert, W. Fürtig \\ Landessternwarte, Königstuhl, D-69117 Heidelberg
}

H. Böhnhardt

Universitätssternwarte, Scheinerstr., D-81679 München

\author{
H. Nicklas \\ Universitätssternwarte, Geismarlandstr., D-37083 Göttingen
}

\begin{abstract}
Focal reducers are nowadays widely used on large telescopes as multi-mode instruments. Some general aspects of the design of focal reducers are discussed. FORS, the focal reducer/low-dispersion spectrograph for the ESO VLT is presented as an example for such a multi-mode instrument on a large telescope.
\end{abstract}

\section{Introduction}

Most of the dark time on large telescopes is used for the observation of faint objects with intensities well below the sky background. The instrumentation for such observations has to be optimized to take full advantage of the telescope's light collecting area. This means not only minimizing the reflection and absorption losses over a broad spectral range and using an efficient detector, but also a high instrument reliability and a fast setup of the desired observing modes.

In recent times, multi-mode instruments for both, imaging and spectroscopy became very popular as they allow to carry out various scientific programs in an efficient way due to their flexibility. The heart of such multi-mode instruments is a focal reducer.

Focal reducers were originally designed to increase the detectability of faint extended sources by decreasing the focal ratio of the telescope (Courtès 1952, Meinel 1956, Courtès 1960). Nowadays, with CCD's as detectors, focal reducers are commonly used on large telescopes.

\section{Focal reducer principle}

A focal reducer consists of a collimator and a camera. The collimator is located behind the telescope's focal plane and produces parallel light. This is reimaged by the camera onto the detector. The flexibility arises (a) from the collimated beam section in which various optical components can be inserted (e.g. filters, Fabry-Perot's, polarization optics and dispersing elements as gratings and/or 
grisms) and (b) from the free access to the focal plane of the telescope which allows to insert e.g. masks, slits and optics for integral field spectroscopy.

It should be noted, that the parallel beam is absolutely necessary only for the dispersing elements. Those would produce severe optical aberrations (mainly astigmatism) if used in an incompletely collimated beam. Thus, any spectrograph is based on the focal reducer principle. Collimators of pure spectrographs are in most cases off-axis paraboloids which suffer from strong aberrations (coma) when used to cover a wide field of view. Therefore, dioptric collimator designs are generally more favourable for multi-mode instruments.

Moreover, as the beam size of faint abject spectrographs is of the order of the detector dimensions (see Table 3), also dioptric camera designs are preferred in such instruments since they allow to use large detectors without vignetting.

\section{Detector matching}

An essential aspect of the design of a focal reducer system is the detector matching with respect to sampling and field of view.

\subsection{Pixel matching}

According to the Whittaker-Shannon sampling theorem each resolution element should be sampled by at least two pixels. Experience shows that a sampling of 2 ...3 pixels is more favourable in most cases (Enard 1984). Therefore, a sampling of 2.5 pixels per resolution element will be adopted in the following. The effective focal ratio $N_{\text {eff }}$ needed to optimize the sampling as a function of the angular spread $\alpha$ of a point source and of the aperture $D$ of the telescope is

$$
N_{e f f}=\frac{2.5 p}{D \tan \alpha}
$$

where $p$ is the pixel size of the detector. This effective focal ratio is calculated for various telescope apertures and seeing values in Table 1 . A pixel size of $24 \mu \mathrm{m}$ was assumed for the calculation.

\begin{tabular}{cccc}
\hline$\alpha$ & & $D$ & \\
$(\operatorname{arcsec})$ & $2.0 \mathrm{~m}$ & $4.0 \mathrm{~m}$ & $8.0 \mathrm{~m}$ \\
\hline 1.0 & 6.2 & 3.1 & 1.5 \\
0.7 & 8.8 & 4.4 & 2.2 \\
0.5 & 12.4 & 6.2 & 3.1 \\
\hline
\end{tabular}

Table 1. Effective focal ratio needed for a sampling of 2.5 pixel for various seeing values $\alpha$ and telescope apertures $D$. Pixel size is assumed as $24 \mu \mathrm{m}$.

Two results can easily be seen: (a) an increase of the telescope diameter leads to faster camera optics for a given seeing. This results in higher complexity of the optics and problems with a possible non-flatness of the CCD and (b) for a given telescope aperture, sampling at optimal seeing leads to slower camera focal ratios and therefore helps for the camera design.

Table 1 gives no information about which seeing value should be chosen for optimum sampling with respect to the detection and observation of faint objects 
which is a main goal for large telescopes. For a given signal-to-noise ratio, the limiting detectable flux for a point source is proportional to $\alpha / D$, assuming an ideal detector. From this it is clear that for the detection of faint sources, good seeing is as important as telescope size. In this sense, the sampling should be optimized for the best values of the seeing expected for some reasonable amount of the time. If the seeing is worse (or for extended objects) the pixels can be binned. In the case of the VLT, the seeing is expected to be better than 0.5 arcsec within $17 \%$ of the time (Swings et al. 1990). Thus the optimum focal ratio would be 3.1 .

The angular spread $\alpha$ of a point source is not only given by the seeing but also by the image quality of the instrument. This implies, that excellent image quality of the instrument is as important as excellent seeing for the observation of faint sources (see e.g. Hubble Space Telescope, where no seeing is present).

\subsection{Field of view}

With a sampling of 2.5 pixels per resolution element as assumed above, the field of view $\Omega$ provided by a detector is given by

$$
\tan \Omega=\frac{\tan \alpha}{2.5} n
$$

which depends only on the seeing value adopted for optimum sampling and the number $n$ of pixels. Thus, for a given seeing, the field of view can only be increased by increasing the number of pixels.

One possibility is the mosaicing of CCD's. For very large telescopes, this leads to problems with the size of the optics required to cover the field, as shown in Table 2, where the linear diameter of the field in the focal plane of the telescope is given for the ESO $3.6 \mathrm{~m}$ and the VLT $8.0 \mathrm{~m}$ telescopes with a $2 \mathrm{k} \times 2 \mathrm{k}$ and a $4 \mathrm{k} \times 4 \mathrm{k}$ CCD (with identical pixel size). A sampling of 2.5 pixel per 0.5 arcsec seeing was assumed for the calculation. For a given $C C D$, the angular field of view is identical for both telescopes, but the linear diameter, which is closely related to the size of the collimator optics, is drastically increased for the VLT.

\begin{tabular}{cccc}
\hline $\begin{array}{c}\text { Number } \\
\text { of pixels }\end{array}$ & Field & \multicolumn{2}{c}{ Linear field diameter } \\
$\left(\right.$ arcmin $\left.^{2}\right)$ & ESO $3.6 \mathrm{~m}$ & VLT $8.0 \mathrm{~m}$ \\
\hline $2 \mathrm{k} \times 2 \mathrm{k}$ & $6.8 \times 6.8$ & $80 \mathrm{~mm}$ & $305 \mathrm{~mm}$ \\
$4 \mathrm{k} \times 4 \mathrm{k}$ & $13.7 \times 13.7$ & $160 \mathrm{~mm}$ & $610 \mathrm{~mm}$ \\
\hline
\end{tabular}

Table 2. Angular and linear field which is covered by a $2 k \times 2 k$ and a $4 \mathrm{k} \times 4 \mathrm{k}$ CCD for two telescopes with a sampling of 2.5 pixels per 0.5 arcsec.

The other possibility for covering large fields is the use of the field slicing technique. Several individual focal reducers are combined to cover the field, each of them having 'normal-sized' optics. In Delabre et al. (1994), a study of a wide field imager spectrograph for the Nasmyth focus of the ESO VLT is described. This instrument consists of 4 folded focal reducers each covering a field of $7 \times 7$ $\operatorname{arcmin}^{2}$. 


\section{Spectroscopy}

\subsection{General aspects}

Focal reducers as faint object spectrographs are usually used for low-dispersion spectroscopy with spectral resolutions of up to some 1000 . The spectral resolution $R$ for a slit width $\phi$ is given by

$$
R=\frac{d}{D \tan \phi}
$$

where $d$ is the diameter of the collimated beam and $D$ is the aperture of the telescope. From this relation it is clear that as the telescope diameter increases, the beam diameter must also increase or the slit width has to be decreased (or some combination of these).

Decreasing the slit width to increase the resolution without losing light is only possible if the seeing is correspondingly good. As discussed above, this also requires a good image quality of the telescope and the focal reducer. It furthermore implies that for low-dispersion spectroscopy (broad spectral range) an atmospheric dispersion corrector (ADC) will be needed.

As dispersing elements in most cases grisms are used. These allow in general for a less complex (straight-through) layout of the instrument and smaller camera optics compared to spectrographs using gratings. Furthermore, the efficiency of a grism is higher than that of a corresponding reflection grating by factors up to 1.2. Also the efficiency in the imaging mode is better as no additional mirror is required. The price to be paid for these advantages is a loss in spectral resolution with respect to reflection gratings which (at the same beam size) can be as high as a factor of 4 . Therefore, the maximum spectral resolution which can be reached with grisms will not be significantely higher than about 1000 .

\subsection{Multi-object spectroscopy}

Going to fainter magnitudes, the number of objects within the field of view of the instrument will be larger than 1 for many classes of objects. In this case, the recording of the spectra of several sources simultaneously will increase the total efficiency of the instrument.

There are several possibilities for realizing a multi-object spectrographic unit depending on the spatial distribution and brightness of the sources. In the following I will concentrate on instruments using masks or slitlets for the selection of the objects.

Table 3 gives a (non-complete) list of such presently available and planned multi-object spectrographs at large telescopes. More information about these instruments can be found e.g. in Dekker et al. (1986) (EMMI), Buzzoni et al. (1984) (EFOSC), LeFèvre et al. (1994) (MOS/SIS), Allington-Smith et al. (1992a) (LDSS2), Oke et al. (1994) (LRIS), Seifert et al. (1994) (FORS), Sasaki et al. (1994) (FOCAS) and Allington-Smith et al. (1992b) (IFOS).

Multi-object spectrographs of this type can cover an intermediate field of view and are especially well suited for the observation of very faint objects, as the sky background can be determined with high accuracy near the object. The slit length necessary will be in the order of 10 to 20 arcsec for point like or only 


\begin{tabular}{|c|c|c|c|c|c|}
\hline Telescope & $\begin{array}{l}\text { Focal } \\
\text { Reducer }\end{array}$ & $\begin{array}{c}\text { Field } \\
\text { angular/linear }\end{array}$ & $\begin{array}{l}\text { Mask } \\
\text { Realiz. }\end{array}$ & $N_{\text {eff }}$ & $\begin{array}{l}\text { Beam } \\
\text { Diam. }\end{array}$ \\
\hline $\mathrm{NTT}_{1}, 3.5 \mathrm{~m}$ & EMMI & $10^{\prime} \times 10^{\prime} / 155 \mathrm{~mm}$ & $P$ & 2.5 & $50 \mathrm{~mm}$ \\
\hline $\mathrm{ESO}, 3.6 \mathrm{~m}$ & EFOSC & $5^{\prime} \times 5^{\prime} / 70 \mathrm{~mm}$ & $\mathbf{P}$ & 2.5 & $40 \mathrm{~mm}$ \\
\hline CFHT, $3.6 \mathrm{~m}$ & MOS/SIS & $10^{\prime} \times 10^{\prime} / 120 \mathrm{~mm}$ & $\mathrm{~L}$ & 2.8 & $46 \mathrm{~mm}$ \\
\hline WHT, $4.2 \mathrm{~m}$ & LDSS2 & $11.5^{\prime} / 155 \mathrm{~mm}$ & $\mathrm{P}$ & 2.0 & $70 \mathrm{~mm}$ \\
\hline KECK, $9.8 \mathrm{~m}$ & LRIS & $6^{\prime} \times 8^{\prime} / 380 \mathrm{~mm}$ & $\mathbf{P}$ & 2.3 & $147 \mathrm{~mm}$ \\
\hline VLT, $8.2 \mathrm{~m}$ & FORS & $6.8^{\prime} \times 6.8^{\prime} / 305 \mathrm{~mm}$ & S & 3.1 & $90 \mathrm{~mm}$ \\
\hline SUBARU, $8.3 \mathrm{~m}$ & FOCAS & $6^{\prime} \times 6^{\prime} / 255 \mathrm{~mm}$ & $\mathbf{S}$ & 3.7 & $100 \mathrm{~mm}$ \\
\hline GEMINI, $8.1 \mathrm{~m}$ & IFOS & $12 ' 170 \mathrm{~mm}$ & $\mathrm{P} / \mathrm{L}$ & 2.0 & $125 \mathrm{~mm}$ \\
\hline
\end{tabular}

Table 3. Basic characteristics of multi-mode instruments capable of multi-object spectroscopy using masks. The technical realization of the mask can be done by punching $(\mathrm{P})$, laser-cutting $(\mathrm{L})$ or individual slitlets (S).

slightly extended objects such as distant galaxies. This allows for a number of 3 to 6 slits per arcmin of the field of view.

Several techniques are available for the production of the aperture masks. Punching machines and laser-cutting devices are used to produce slits in thin sheets of metal. While this allows for slits of arbitrary shape, real on-line production of the masks is difficult. This is due to the limited space available at the telescope's focal plane which prevents the implementation of the mask producing device within the main instrument. In this respect, multi-slit units, consisting of several pairs of individually moveable slitlets to form the slits are more favourable, as they can be completely integrated in the instrument. As mechanical rigidity of the slitlets is required, their efficient use is restricted to large telescopes with long focal lengths to provide slits with reasonable angular length on the sky.

\section{Example: Spectroscopy with FORS}

As an example for such a multi-mode instrument, the expected spectroscopic performance of FORS, the FOcal Reducer/low-dispersion Spectrograph for the ESO VLT, will be described in the following.

\subsection{Basic characteristics}

FORS is an all dioptric instrument designed for direct imaging, low-dispersion multi-object spectroscopy, imaging polarimetry and spectro-polarimetry of faint objects in the wavelength range from $330 \mathrm{~nm}$ to $1.1 \mu \mathrm{m}$. The field of view is $6.8 \times 6.8 \mathrm{arcmin}^{2}$ with an image scale of $0.2 \mathrm{arcsec} / \mathrm{pixel}$. A configuration with an image scale of 0.1 arcsec per pixel can be selected by remote exchange of the collimator to take full advantage of periods of excellent seeing. FORS will concentrate more than $80 \%$ of the light of a point object within 1 pixel nearly all over the field in both configurations.

The image motion due to flexure of the instrument is calculated to be less than a quarter of a pixel during two hours of observation. This small amount is not due to the overall stiffness of the FORS structure, but is reached by means of a flexure compensation. A schematic view of the opto-mechanical layout is shown in Fig. 1. Two copies of the instrument are under construction and will 


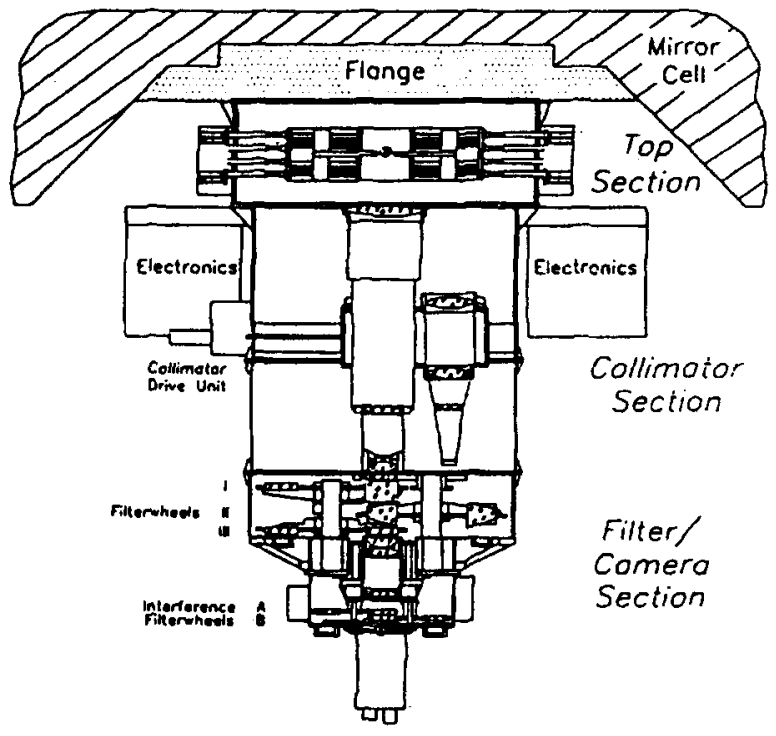

Figure 1. Schematic view of the opto-mechanical layout of FORS

be installed at the Cassegrain foci of the VLT unit telescopes Nos. 1 and 3 . More details on FORS can be found in Seifert et al. (1994).

\subsection{Spectroscopy}

Both copies of FORS will be equiped with a set of six 'standard' (i.e. working in first order) grisms with reciprocal dispersions of 45 to $230 \AA / \mathrm{mm}$. The spectral resolution will be 1200 at $H_{\alpha}$ with a slit width of 1 arcsec. For the second copy of the instrument, a medium dispersion mode is presently under investigation: with grisms working in an echelle mode, reciprocal dispersions of 13 to $35 \AA / \mathrm{mm}$ and a spectral resolution of up to $3000\left(H_{\alpha}, 1\right.$ arcsec slit) will be reached. For more details on the design of the grisms see Fürtig and Seifert (1994).

Operation under full remote control, the minimization of human intervention and the possiblity of flexible scheduling of FORS, as well as a high spectrophotometric accuracy for the observation of faint sources were main requirements for the design of the instrument.

This led to a multi-object spectroscopy unit which is based on a slitlet design with 19 pairs of individually moveable slitlets. The slit length is fixed to 22.5 arcsec projected onto the sky. The slit width can be continuously adjusted down to less than 0.1 arcsec and the complete field of view can be used. A schematic top view of the unit is shown in Fig. 2.

With this design, a high quality of the slitblades - critical for the calibration - is possible. Also both, the slit position in the field and the slit width can be reproduced to better than a tenth of a pixel. The total set-up time of any slit configuration is only about 20 seconds as each slitlet has its own drive. The unit is completely self-contained, thus a direct set-up from the workstation, as well as 


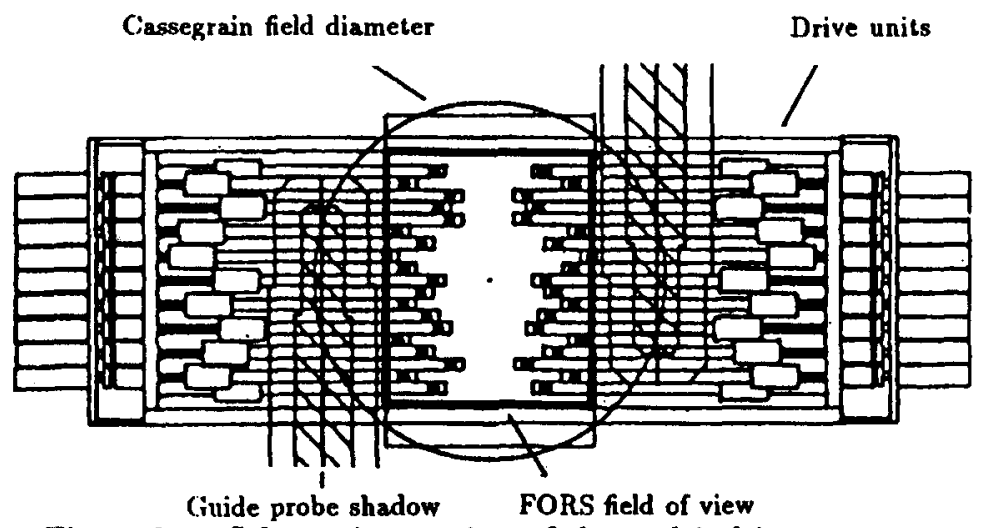

Figure 2. Schematic top view of the multi-object spectroscopy unit of FORS. The 19 slitlet pairs cover the FORS field of view. The total field at the Cassegrain focus is indicated by the circle. Also two possible locations of the shadow of the guide probe of the VLT are indicated (hatched areas).

the on-line adaptation of the slit width and position to varying seeing conditions and/or zenith distance etc. is possible. Dedicated software will allow to make efficient use of the multi-object spectroscopy mode. A more detailed technical description of the multi-object spectroscopy unit can be found in Mitsch et al. (1994).

The slits will also be used to provide a mask for imaging polarimetry of extended objects. A separate long slit mask with 9 slits of fixed slit width is included in the unit.

The limiting magnitudes $\left(m_{B}\right)$ will be about $24 \mathrm{mag}$ for low-dispersion spectroscopy and 20 mag for spectro-polarimetry for an observation of half an hour.

Acknowledgments. We thank the whole FORS team for their hard work to transform a concept to a final design. The FORS project is performed under ESO contract 37458/ESO/VLT/91/7844/GWI and supported by the German Federal Ministry of Science and Technology with ID-Nos. 052HD50A, 052GO20A and $052 \mathrm{MU} 104$.

\section{Discussion}

N. Douglas: Please comment on the active (?) stabilisation required to overcome the stiffness problem.

W. Seifert: The flexure compensation mentioned in my talk is a purely passive system based on fact that an image motion caused by a decentering of the collimator due to flexure is compensated by a corresponding image motion due 
to a tilt of the camera. During the test phase of the instrument, this camera tilt can be fine adjusted to minimize residual image motion.

G. Cecil: 1.) What are you doing for ADC ? 2.) How large are the PSF variations across the field of view ? 3.) What is being done about ghosts ?

W. Seifert: An ADC will be implemented by ESO for the Cassegrain focus of the VLT. This will allow to observe up to zenith distances of about 50 degrees without significant loss of image quality. Concerning your second question, the PSF will vary significantly across the field of view by up to $50 \%$. This value is partly due to the PSF variation of the telescope itself. But I think that for most applications, the reproducability of the PSF is more critical and here, the variations are estimated to be less than $5 \%$. To the third part of your question: a detailed ghost image analysis was carried out. The relative peak intensity of ghosts will be less than $10^{-4}$. Special care was taken to minimize the so-called sky concentration, which was calculated to be less than $1 \%$.

R.Bacon: Do you expect this instrument to be competitive with e.g. MOS/SIS, with only 19 spectra compared to say 100 for MOS ?

$W$. Seifert: If you are interested in the whole free spectral range provided by the grism used, the maximum number of spectra obtainable with MOS will be 40 to 60 for a slit length of 10 to 15 arcsec. As mentioned in my talk, the design of the FORS multi-object spectroscopy unit was mainly driven by the requirement to have a completely self-contained unit allowing spectrophotometry of faint objects with high accuracy. This led to the slitlet design with fixed slit length. There will surely be some statistical programs for which not only a larger number of slits, but also a larger field of view would be favourable. But I think that FORS will be very effective for the observation of individual or small groups of objects.

$K$. Jockers: Is the design of FORS made for active seeing compensation at the $8 \mathrm{~m}$ telescope?

W. Seifert: As the maximum field which can be supported by adaptive optics (about 20 arcsec) is much smaller than the FORS field of view, this is not foreseen at the present time.

\section{References}

Allington-Smith, J., Breare, M., Ellis, R., Gellatly, D., Glazebrook, K., Jorden, P., MacLean, J., Oates, P., Shaw, G., Tanvir, N., Taylor, K., Taylor, P., Webster, J., \& Worswick, S. 1992a, in ESO Conf. on Progress in Telescope and Instrumentation Technologies, M.-H. Ulrich (ed.), 1992, 671

Allington-Smith, J., Worswick, S., \& Gellatly, D. 1992b, in ESO Conf. on Progress in Telescope and Instrumentation Technologies, M.-H. Ulrich (ed.), 1992, 605

Buzzoni, B., Delabre, B., Dekker, H., D'Odorico, S., Enard, D., Focardi, P., Gustafsson, B., Nees, W., Paureau, J., \& Reiss, R. 1984, The Messenger, $1984,38,9$

Courtès, G. 1952, C.R. Acad. Sciences, 1952, 234, 506 
Courtès, G. 1960, Ann. d'Astrophys., 1960, 23, 115

Dekker, H., Delabre, B., \& D'Odorico, S. 1986, in Proc. SPIE 627, 339

Delabre, B., D'Odorico, S., \& Vettolani, P. 1994, in Proc. SPIE 2198, D.L. Crawford and E.R. Craine (eds.), 1994, in press

Enard, D. 1984, in IAU Colloquium No. 79, Very Large Telescopes, their Instrumentation and Programs, M.-H. Ulrich and K. Kjär (eds.), 1984, 439

Fürtig, W., \& Seifert, W. 1994, 'A Set of Grisms for FORS', in these proceedings

LeFèvre, O., Crampton, D., Felenbok, P., \& Monnet, G. 1994, Astron. Astrophys., 1994, 282, 325

Meinel, A.B. 1956, ApJ, 1956, 124, 652

Mitsch W., Rupprecht G., Seifert W., Nicklas H., \& Kiesewetter S. 1994, in Proc. SPIE 2198, D.L. Crawford and E.R. Craine (eds.), 1994, in press

Oke, J.B., Cohen, J., Carr, M., Cromer, J., Dingizian, A., Harris, F.H., Lucinio, R., Labrecque, S., Schaal, W., \& Southard, S. 1994, in Proc. SPIE 2198, D.L. Crawford and E.R. Craine (eds.), 1994, in press

Swings, J.-P., Appenzeller, I., Ardeberg, A., Charvin, P., Lelièvre, G., Perrier, C., Sarazin, M., Schuster, H.-E., \& Shaver, P. 1990, in ESO VLT Report No. 62, VLT Site Selection Working Group Final Report, M. Sarazin (ed.), 1990, 134

Sasaki, T., Iye, M., Yamashita, T., \& Shibata, T. 1994, in Proc. SPIE 2198, D.L. Crawford and E.R. Craine (eds.), 1994, in press

Seifert, W., Mitsch, W., Nicklas, H., \& Rupprecht, G. 1994, in Proc. SPIE 2198, D.L. Crawford and E.R. Craine (eds.), 1994, in press 